\title{
Glucose Control and Weight Change Associated with Treatment with Exenatide Compared with Basal Insulin: A Retrospective Study
}

\author{
Christopher Ll. Morgan (D) Q Qing Qiao · Susan Grandy • \\ Kristina Johnsson · Sara Jenkins-Jones · Sarah Holden • \\ Craig J. Currie
}

Received: October 30, 2017 / Published online: January 9, 2018

(c) The Author(s) 2018. This article is an open access publication

\section{ABSTRACT}

Introduction: The aim of the study was to compare glycemic and weight change outcomes for type 2 diabetes patients treated with either exenatide once-weekly (EQW) or exenatide twice-daily (EBID) with those patients treated with basal insulin (BI).

Methods: Retrospective data (2010-2014) were extracted from the Clinical Practice Research Datalink, a UK primary care database. Patients previously naïve to injectable therapy initiating EQW, EBID, or BI were extracted and matched by propensity score within two analyses (EQW vs $\mathrm{BI}$ and EBID vs BI). Absolute and relative change in HbA1c and weight from baseline and the proportion of patients achieving HbA1c $\leq 7.0 \%(53 \mathrm{mmol} / \mathrm{mol})$ combined with weight reduction targets of (1) any weight loss or $(2) \geq 5.0 \%$ from baseline were compared at 6 and $12-24$ months.

Enhanced content To view enhanced content for this article go to http://www.medengine.com/Redeem/ 890DF0606E7785B5.

C. Ll. Morgan ( $\square)$. S. Jenkins-Jones · S. Holden .

C. J. Currie

Pharmatelligence, Cardiff, UK

e-mail: Chris.morgan@pharmatelligence.co.uk

Q. Qiao · K. Johnsson

AstraZeneca, Gothenburg, Sweden

S. Grandy

AstraZeneca, Gaithersburg, USA
Results: A total of 485 patients initiated EQW, 3573 EBID, and 13,503 BI. In the propensity matched EQW versus BI analysis, mean HbA1c decreased with changes of $-1.33 \%(-14.5$ $\mathrm{mmol} / \mathrm{mol})$ and $-1.24 \%(-13.5 \mathrm{mmol} / \mathrm{mol})$ at 6 months and $-1.19 \%(-13.0 \mathrm{mmol} / \mathrm{mol})$ and $-1.17 \%(-12.8 \mathrm{mmol} / \mathrm{mol})$ at $12-24$ months, respectively. Respective weight change was $-3.7 \mathrm{~kg}$ versus $+1.2 \mathrm{~kg}(p<0.001)$ and $-3.2 \mathrm{~kg}$ versus $+2.5 \mathrm{~kg}(p<0.001)$. Significantly more EQW patients achieved the combined HbA1c $\leq 7.0 \%(53 \mathrm{mmol} / \mathrm{mol})$ and weight loss target $(22.4 \%$ versus $9.9 \%$ at 6 months and $18.2 \%$ versus $8.0 \%$ at $12-24$ months, respectively) and $\mathrm{HbA} 1 \mathrm{c} \leq 7.0 \% \quad(53 \mathrm{mmol} / \mathrm{mol})$ and minimum $5 \%$ weight loss $(11.8 \%$ versus $3.7 \%$ at 6 months, and $8.0 \%$ versus $0.0 \%$ at 12-24 months). For EBID versus BI, similar results were found.

Conclusion: In this real-world data analysis, exenatide QW and exenatide BID were associated with similar glycemic control and greater weight reduction compared with basal insulin.

Keywords: Exenatide; Basal insulin

\section{INTRODUCTION}

Most commonly, patients diagnosed with type 2 diabetes (T2DM) are initially treated with lifestyle modification, focussing upon diet and exercise regimens. If this is unsuccessful, 
patients are then treated with glucose-lowering drugs. Current guidelines recommend that patients initiate treatment with metformin, assuming an absence of any contraindications [1]. If and when their glucose control worsens, treatment is intensified with the addition of other glucose-lowering drugs in combination as dual-therapy, and then as triple therapy. Ultimately patients may require insulin. The treatment of T2DM, however, is complex, as whilst the primary aim of treatment is to maintain normoglycemia, this must be balanced against other factors such as weight gain and hypoglycemia resulting from exposure to either insulin secretagogues or exogenous insulin injections. This is particularly important as it has been reported that over $80 \%$ of patients are overweight or obese on first T2DM presentation [2] and some antidiabetic drugs are associated with weight gain [3] which is known to increase cardiovascular risk.

During the 2000s, incretin-based drugs became available, which provided new treatment options for patients with T2DM. However, these new therapies are more expensive than established agents [4] and so need to demonstrate not only efficacy but also their relative cost-effectiveness. Exenatide affects the incretin pathway and is part of the glucagon-like peptide-1 receptor agonist (GLP-1RA) class. Like insulin, it is injectable, but has the benefit that a recent formulation with microsphere technology, exenatide QW (Bydureon), only has to be administered once weekly with fixed dose, thus removing the need to titrate. Randomized controlled trial (RCT) data has shown at least equivalence in glucose control [5-7] for patients treated with exenatide formulations compared with insulin-based regimens and also significant reductions in weight $[5,7]$. Additionally, one real-world analysis has shown that exenatide BID conferred cardiovascular benefit when compared to insulin-based regimens [8], and though the results were not statistically significant, there was an association with reduced mortality [9]. A potentially key contributing factor could be that exenatide is associated with weight loss [10, 11]. In addtion, exenatide avoids the need to expose people to exogenous insulin which has been reported to increase the risk of serious adverse outcomes [12]. A study comparing exenatide QW to placebo reported a non-significant reduction in rate of major adverse cardiovascular event (MACE) [13].

In this study, we compared real-world effectiveness outcomes for patients with type 2 diabetes initiating glucose-lowering treatment with either exenatide as a weekly formulation (exenatide QW) or as a twice-daily formulation (exenatide BID) versus patients initiating treatment with basal insulin-based regimens.

\section{METHODS}

\section{Data Source}

Patients were selected from the Clinical Practice Research Datalink (CPRD) [14] between January 1, 2009 and December 31, 2014. CPRD is a longitudinal, anonymized research database derived from nearly 700 primary care practices in the UK which are considered representative of UK primary care as a whole. The primary care dataset (CPRD GOLD) comprises data on demographics, diagnoses, hospital referrals, prescriptions emanating in primary care, and other aspects of patient care. Diagnostic information in the CPRD primary care dataset is recorded using the Read code classification, a UK primary care practice standard. This article is based on previously conducted studies and does not involve any new studies of human or animal subjects performed by any of the authors.

\section{Patient Selection}

Patients defined by CPRD as being of acceptable research quality were classified as having type 2 diabetes if they had a Read code indicative of diabetes and if at least one of the following selection criteria applied:

1. More than one diagnostic record exclusively for type 2 diabetes

2. Prescriptions for two or more different classes of non-insulin glucose-lowering therapy

3. A diagnostic code indicative of type 2 diabetes (regardless of conflicting diagnoses of type 1 or nonspecific diabetes) plus a 
prescription for a non-insulin glucose lowering therapy

Cases were defined as those patients prescribed exenatide. Two groups of cases were selected on the basis of the exenatide formulation received, that is either exenatide QW (Bydureon), a onceweekly formulation or exenatide BID (Byetta), a twice-daily formulation. Exenatide could be prescribed either as monotherapy or in combination with one or more other oral glucoselowering therapies. Patients were excluded if they had received prior injectable diabetes therapy or had less than 90 days of continuous exposure to the exenatide therapy.

Control patients were selected from the pool of type 2 diabetes patients who had a prescription for basal insulin either as monotherapy or in combination with one or more other oral glucose-lowering therapies. The same exclusion criteria applied to exenatide cases were used.

Patients in each case group (exenatide QW and exenatide BID) were matched by propensity score. The covariates included in the propensity matching were age, gender, BMI, diabetes duration, year of index exposure, HbA1c, smoking status, systolic blood pressure, total cholesterol, serum creatinine, and Charlson index [15]. The Charlson index predicts 12-month mortality based on the presence of 22 underlying morbidities. To maximize patient numbers with data available at each time point, patients were rematched for each outcome.

Date of first prescription of either exenatide or basal insulin was defined as the index date.

\section{Outcomes}

The primary outcomes were $\mathrm{HbA1c}$ and weight change. Baseline measures were defined as any measurement between - 180 days and index date. Change in each outcome was measured from baseline to the nearest recorded measurement at 6 months post-baseline ( \pm 90 days), and $12-24$ months post-baseline ( \pm 90 days) for those patients remaining on their index regimen. For HbA1c, change was measured as absolute difference and proportion of patients for whom HbA1c fell below $7.0 \%$
(53 mmol/mol). Weight change was recorded as absolute change in kilograms and as percentage change. Two additional composite endpoints categorized patients that achieved HbA1c $\leq 7.0 \%(53 \mathrm{mmol} / \mathrm{mol})$ and also (a) any weight loss and (b) weight loss of $\geq 5 \%$ baseline.

A secondary outcome was time to severe hypoglycemic event defined by Read code in the primary care dataset. Time to event was compared in a Cox proportional hazard model adjusting for age, gender, baseline $\mathrm{HbA1c}$, and Charlson index.

\section{Data and Statistical Analysis}

Baseline characteristics of patients initiated on either exenatide BID or exenatide QW and basal insulin were presented. Differences between characteristics were tested using the $t$ test for continuous variables and Pearson $\chi^{2}$ for categorical variables.

Changes in $\mathrm{HbA} 1 \mathrm{c}$ and weight from baseline at 6 and 12-24 months were calculated and differences between treatment groups compared. Differences were considered as both an absolute and relative change from baseline and compared between groups using the $t$ test.

Two composite endpoints were considered that were based on the proportion of patients:

(a) Reaching a target of HbA1c $\leq 7.0 \%$ $(53 \mathrm{mmol} / \mathrm{mol})$ with any weight reduction

(b) Reaching a target of HbA1c $\leq 7.0 \%$ $(53 \mathrm{mmol} / \mathrm{mol})$ with weight reduction $\geq 5 \%$ The proportions of patients reaching these endpoints were compared by $\chi^{2}$ test.

Analyses were performed for all patients and for those matched by propensity score.

\section{RESULTS}

\section{Study Numbers and Baseline Characteristics}

Following the application of the study criteria, the study cohorts contained 485 patients initiating on exenatide QW, 3573 patients initiating exenatide BID, and 13,503 initiating basal insulin. The overall baseline characteristics are 
Table 1 Baseline characteristics for patients treated with exenatide QW versus basal insulin

\begin{tabular}{|c|c|c|c|c|c|}
\hline \multirow[b]{2}{*}{ Number of patients } & \multicolumn{2}{|c|}{ Exenatide QW } & \multicolumn{2}{|c|}{ Basal insulin } & \multirow[t]{2}{*}{$p$ value } \\
\hline & 485 & & 13,503 & & \\
\hline Female $[n(\%)]$ & 206 & $(42.5 \%)$ & 5913 & $(43.8 \%)$ & 0.576 \\
\hline Age, years [mean, $(\mathrm{sd})]$ & 57 & $(11.3)$ & 64.6 & $(15.2)$ & $<0.001$ \\
\hline Diabetes duration, years [mean, (sd)] & 8.5 & $(5.2)$ & 10.2 & $(8.0)$ & $<0.001$ \\
\hline Follow-up time, months [mean, (sd)] & 11.3 & $(9.1)$ & 9.6 & $(11.0)$ & $<0.001$ \\
\hline BMI, [mean, (sd)] & 37.5 & $(6.7)$ & 29.9 & (6.3) & $<0.001$ \\
\hline HbAlc DCCT, \% [mean, (sd)] & 9.4 & $(1.7)$ & 9.8 & $(2.0)$ & $<0.001$ \\
\hline HbAlc IFCC [mean, (sd)] & 79.3 & $(18.6)$ & 83.2 & $(22.1)$ & $<0.001$ \\
\hline Serum creatinine $\mu \mathrm{mol} / \mathrm{l}[\mathrm{mean},(\mathrm{sd})]$ & 76.6 & $(21.2)$ & 97.8 & $(49.4)$ & $<0.001$ \\
\hline Systolic blood pressure, mmHg [mean, (sd)] & 133.6 & $(14.6)$ & 132.5 & $(17.2)$ & 0.124 \\
\hline Diastolic blood pressure, $\mathrm{mmHg}$ [mean, $(\mathrm{sd})]$ & 78.2 & $(9.3)$ & 75.3 & $(10.4)$ & $<0.001$ \\
\hline Total cholesterol, mmol/l [mean, (sd)] & 4.4 & $(1.1)$ & 4.4 & $(1.4)$ & 0.516 \\
\hline Charlson index [mean, (sd)] & 2.3 & $(1.3)$ & 3.3 & $(2.1)$ & $<0.001$ \\
\hline Primary care contacts in previous 12 months [mean, (sd)] & 11.2 & $(11.2)$ & 11.1 & $(11.4)$ & 0.897 \\
\hline Prior MACE (\%) & 44 & $(9.1 \%)$ & 2402 & $(17.8 \%)$ & $<0.001$ \\
\hline Prior cancer $(\%)$ & 23 & $(4.7 \%)$ & 1754 & $(13.0 \%)$ & $<0.001$ \\
\hline \multicolumn{6}{|l|}{ Smoking history } \\
\hline Never smoked & 209 & $(43.1 \%)$ & 5741 & $(42.5 \%)$ & 0.218 \\
\hline Ex-smoker & 202 & $(41.6 \%)$ & 5234 & $(38.8 \%)$ & \\
\hline Current smoker & 71 & $(14.6 \%)$ & 2339 & $(17.3 \%)$ & \\
\hline \multicolumn{6}{|l|}{ Concomitant therapy } \\
\hline Study drug monotherapy & 55 & $(11.3 \%)$ & 3306 & $(24.5 \%)$ & \\
\hline Metformin, sulfonylurea & 160 & $(33.0 \%)$ & 2658 & $(19.7 \%)$ & \\
\hline Sulfonylurea & 33 & $(6.8 \%)$ & 1632 & $(12.1 \%)$ & \\
\hline Metformin & 126 & $(26.0 \%)$ & 1629 & $(12.1 \%)$ & \\
\hline Other insulin & 0 & $(0.0 \%)$ & 1461 & $(10.8 \%)$ & \\
\hline Metformin, other insulin & 2 & $(0.4 \%)$ & 656 & $(4.9 \%)$ & \\
\hline Metformin, sulfonylurea, DPP4 & 32 & $(6.6 \%)$ & 574 & $(4.3 \%)$ & \\
\hline Sulfonylurea, DPP4 & 4 & $(0.8 \%)$ & 291 & $(2.2 \%)$ & \\
\hline Metformin, sulfonylurea, TZD & 9 & $(1.9 \%)$ & 247 & $(1.8 \%)$ & \\
\hline Metformin, DPP4 & 25 & $(5.2 \%)$ & 225 & $(1.7 \%)$ & \\
\hline DPP4 & 5 & $(1.0 \%)$ & 136 & $(1.0 \%)$ & \\
\hline Metformin, TZD & 19 & $(3.9 \%)$ & 124 & $(0.9 \%)$ & \\
\hline
\end{tabular}


Table 1 continued

\begin{tabular}{|c|c|c|c|c|c|}
\hline \multirow[b]{2}{*}{ Sulfonylurea, TZD } & \multicolumn{2}{|c|}{ Exenatide $Q W$} & \multicolumn{2}{|c|}{ Basal insulin } & \multirow[t]{2}{*}{$p$ value } \\
\hline & 1 & $(0.2 \%)$ & 106 & $(0.8 \%)$ & \\
\hline Metformin, sulfonylurea, other insulin & 0 & $(0.0 \%)$ & 56 & $(0.4 \%)$ & \\
\hline TZD & 2 & $(0.4 \%)$ & 45 & $(0.3 \%)$ & \\
\hline Sulfonylurea, other insulin & 0 & $(0.0 \%)$ & 34 & $(0.3 \%)$ & \\
\hline Glinide & 0 & $(0.0 \%)$ & 26 & $(0.2 \%)$ & \\
\hline Metformin, TZD, other insulin & 0 & $(0.0 \%)$ & 22 & $(0.2 \%)$ & \\
\hline Other & 12 & $(2.5 \%)$ & 275 & $(2.0 \%)$ & \\
\hline
\end{tabular}

shown in Table 1 for exenatide QW versus basal insulin and Table 2 for exenatide BID versus basal insulin. Compared with those initiating basal insulin, those initiating exenatide QW were younger (57.0 versus 64.6 years), had higher BMI (37.5 versus 29.9) and lower HbA1c $[9.4 \% \quad(79.2 \mathrm{mmol} / \mathrm{mol}) \quad$ versus $9.8 \%$ $(84.7 \mathrm{mmol} / \mathrm{mol})]$. The patients also had lower co-morbidity indicated by a lower Charlson Index (2.3 versus 3.3) and prior history of MACE (9.1\% versus $17.8 \%)$ and cancer $(4.7 \%$ versus $13.0 \%)$. Similar differences appeared in those initiating exenatide BID. The numbers of patients in each propensity matched analysis are shown in Tables 4 and 5.

\section{Exenatide QW Versus Basal Insulin: HbA1c Change}

In the unmatched cohorts, mean $\mathrm{HbA1c}$ at 6 months fell by $1.3 \%$ for those treated with exenatide QW compared with $1.5 \%$ for those treated with basal insulin. At 12-24 months, the corresponding figures were $1.2 \%$ and $1.5 \%$ (Table 3). In the propensity matched analysis, the mean $\mathrm{HbA1c}$ fell for both treatment groups with respective changes of $1.3 \%$ and $1.2 \%$ at 6 months and $1.2 \%$ and $1.2 \%$ at $12-24$ months. There was no difference in the change in HbA1c between the two treatment groups at the conventional level of statistical significance (Table 4).

\section{Exenatide QW Versus Basal Insulin: Weight Change}

In the unmatched cohorts, mean weight at 6 months fell $3.8 \mathrm{~kg}$ in those patients treated with exenatide QW compared with an increase of $1.3 \mathrm{~kg}$ for those treated with basal insulin, a difference of $5.1 \mathrm{~kg}$. At $12-24$ months the respective figures were -4.0 and $+2.2 \mathrm{~kg}$ (Table 3$)$. In the propensity matched cohorts, mean weight change at 6 months was $-3.7 \mathrm{~kg}$ for patients treated with exenatide QW compared with $+1.2 \mathrm{~kg}$ for those treated with basal insulin; this was a statistically significant difference in weight loss between the groups $(p<0.001)$. Similarly, at $12-24$ months, weight change was $-3.2 \mathrm{~kg}$ in those patients treated with exenatide QW compared with $+2.5 \mathrm{~kg}$ for those treated with basal insulin $(p<0.001$; Table 4).

\section{Exenatide QW Versus Basal Insulin: Combined Endpoint}

In the propensity matched analysis comparing exenatide QW and basal insulin, a significantly greater proportion of patients treated with exenatide QW reached the target of HbA1c $\leq 7.0 \%$ (53 mmol $/ \mathrm{mol}$ ) with corresponding weight loss. The respective proportions at the 6-month and the 12-24-month time windows were $22.4 \%$ versus $9.9 \%$ and $18.2 \%$ versus $8.0 \%$, respectively for exenatide QW and basal insulin. For the 
Table 2 Baseline characteristics for patients treated with exenatide BID versus basal insulin

\begin{tabular}{|c|c|c|c|c|c|}
\hline \multirow[b]{2}{*}{ Number of patients } & \multicolumn{2}{|c|}{ Exenatide BID } & \multicolumn{2}{|c|}{ Basal insulin } & \multirow[t]{2}{*}{$p$ value } \\
\hline & 3573 & & 13,503 & & \\
\hline Female $[n(\%)]$ & 1627 & $(45.5 \%)$ & 5913 & $(43.8 \%)$ & 0.063 \\
\hline Age, years [mean, $(\mathrm{sd})]$ & 56.6 & $(10.6)$ & 64.6 & $(15.2)$ & $<0.001$ \\
\hline Diabetes duration, years [mean, (sd)] & 7.9 & $(4.8)$ & 10.2 & $(8.0)$ & $<0.001$ \\
\hline Follow-up time, months [mean, (sd)] & 11.5 & 11.7 & 9.6 & $(11.0)$ & $<0.001$ \\
\hline BMI, [mean, (sd)] & 38.7 & $(6.7)$ & 29.9 & $(6.3)$ & $<0.001$ \\
\hline HbAlc DCCT, \% [mean, (sd)] & 9.2 & $(1.6)$ & 9.8 & $(2.0)$ & $<0.001$ \\
\hline HbAlc IFCC [mean, (sd)] & 77 & $(17.8)$ & 83.2 & $(22.1)$ & $<0.001$ \\
\hline Serum creatinine $\mu \mathrm{mol} / \mathrm{l}[\mathrm{mean},(\mathrm{sd})]$ & 79.3 & $(22.5)$ & 97.8 & $(49.4)$ & $<0.001$ \\
\hline Systolic blood pressure, $\mathrm{mmHg}$ [mean, (sd)] & 134.5 & $(14.8)$ & 132.5 & $(17.2)$ & $<0.001$ \\
\hline Diastolic blood pressure, $\mathrm{mmHg}$ [mean, $(\mathrm{sd})]$ & 79.2 & $(9.3)$ & 75.3 & $(10.4)$ & $<0.001$ \\
\hline Total cholesterol, mmol/l [mean, (sd)] & 4.3 & $(1.1)$ & 4.4 & $(1.4)$ & $<0.001$ \\
\hline Charlson Index [mean, (sd)] & 2.3 & $(1.4)$ & 3.3 & $(2.1)$ & $<0.001$ \\
\hline Primary care contacts in previous 12 months [mean, (sd)] & 10.8 & $(9.1)$ & 11.1 & $(11.4)$ & 0.145 \\
\hline Prior MACE (\%) & 312 & $(8.7 \%)$ & 2402 & $(17.8 \%)$ & $<0.001$ \\
\hline Prior cancer $(\%)$ & 188 & $(5.3 \%)$ & 1754 & $(13.0 \%)$ & $<0.001$ \\
\hline \multicolumn{6}{|l|}{ Smoking history } \\
\hline Never smoked & 1438 & $(40.2 \%)$ & 5741 & $(42.5 \%)$ & $<0.001$ \\
\hline Ex-smoker & 1574 & $(44.1 \%)$ & 5234 & $(38.8 \%)$ & \\
\hline Current smoker & 545 & $(15.3 \%)$ & 2339 & $(17.3 \%)$ & \\
\hline Concomitant therapy & 16 & & 189 & & \\
\hline Study drug monotherapy & 480 & $(13.4 \%)$ & 3306 & $(24.5 \%)$ & \\
\hline Metformin, sulfonylurea & 1283 & $(35.9 \%)$ & 2658 & $(19.7 \%)$ & \\
\hline Sulfonylurea & 239 & $(6.7 \%)$ & 1632 & $(12.1 \%)$ & \\
\hline Metformin & 942 & $(26.4 \%)$ & 1629 & $(12.1 \%)$ & \\
\hline Other insulin & 5 & $(0.1 \%)$ & 1461 & $(10.8 \%)$ & \\
\hline Metformin, other insulin & 8 & $(0.2 \%)$ & 656 & $(4.9 \%)$ & \\
\hline Metformin, sulfonylurea, DPP4 & 80 & $(2.2 \%)$ & 574 & $(4.3 \%)$ & \\
\hline Sulfonylurea, DPP4 & 17 & $(0.5 \%)$ & 291 & $(2.2 \%)$ & \\
\hline Metformin, sulfonylurea, TZD & 195 & $(5.5 \%)$ & 247 & $(1.8 \%)$ & \\
\hline Metformin, DPP4 & 64 & $(1.8 \%)$ & 225 & $(1.7 \%)$ & \\
\hline DPP4 & 13 & $(0.4 \%)$ & 136 & $(1.0 \%)$ & \\
\hline Metformin, TZD & 149 & $(4.2 \%)$ & 124 & $(0.9 \%)$ & \\
\hline
\end{tabular}


Table 2 continued

\begin{tabular}{|c|c|c|c|c|c|}
\hline \multirow[b]{2}{*}{ Sulfonylurea, TZD } & \multicolumn{2}{|c|}{ Exenatide BID } & \multicolumn{2}{|c|}{ Basal insulin } & \multirow[t]{2}{*}{$p$ value } \\
\hline & 32 & $(0.9 \%)$ & 106 & $(0.8 \%)$ & \\
\hline Metformin, sulfonylurea, other insulin & 0 & $(0.0 \%)$ & 56 & $(0.4 \%)$ & \\
\hline TZD & 26 & $(0.7 \%)$ & 45 & $(0.3 \%)$ & \\
\hline Sulfonylurea, other insulin & 0 & $(0.0 \%)$ & 34 & $(0.3 \%)$ & \\
\hline Glinide & 6 & $(0.2 \%)$ & 26 & $(0.2 \%)$ & \\
\hline Metformin, TZD, other insulin & 1 & $(0.0 \%)$ & 22 & $(0.2 \%)$ & \\
\hline Other & 33 & $(0.9 \%)$ & 275 & $(2.0 \%)$ & \\
\hline
\end{tabular}

second target of $\mathrm{HbA} 1 \mathrm{c} \leq 7.0 \%(53 \mathrm{mmol} / \mathrm{mol})$ and a minimum of $5 \%$ weight loss, there were also a significantly greater proportion of patients achieving the target at each time window with exenatide QW than with BI. The proportions were $11.8 \%$ versus $3.7 \%$, and $8.0 \%$ versus $0.0 \%$ of patients, respectively (Table 4 ).

\section{Exenatide QW Versus Basal Insulin: Time to Severe Hypoglycemia}

There were no severe hypoglycemic events recorded for patients treated with exenatide QW. For patients treated with basal insulin there were 167 events [15.5 per thousand patient years (pky)] in the unmatched cohort and 1 (3.6 pky) in those matched by propensity score (Table 6).

\section{Exenatide BID Versus Basal Insulin: HbA1c}

In the unmatched analysis, HbA1c fell by a mean of $0.9 \%$ in those treated with exenatide BID compared with $1.5 \%$ in those treated with basal insulin at 6 months, and $0.9 \%$ and $1.5 \%$ at 12-24 months (Table 3). In the propensity matched analysis comparing exenatide BID and basal insulin, mean HbA1c fell for both treatment groups with respective changes of $1.0 \%$ and $1.0 \%$ at 6 months, and $1.0 \%$ and $0.9 \%$ at 12-24 months. There was no difference in the change in HbA1c between the two treatment groups at either time point at the conventional level of statistical significance (Table 5).

\section{Exenatide BID Versus Basal Insulin: Weight Change}

At 6 months in the unmatched analysis, mean weight fell by a mean of $4.1 \mathrm{~kg}$ in those treated with exenatide BID compared with a gain of $1.3 \mathrm{~kg}$ in those treated with basal insulin. At 12 months the respective figures were -5.3 and $+2.2 \mathrm{~kg}$ (Table 3 ). In the propensity matched analysis comparing exenatide BID and basal insulin, mean weight fell for those prescribed exenatide BID but increased for those prescribed basal insulin. The respective changes were -3.5 and $+0.82 \mathrm{~kg}$ at 6 months and -4.7 and $+1.7 \mathrm{~kg}$ at 12-24 months. All differences were statistically significant (Table 5).

\section{Exenatide BID Versus Basal Insulin: Combined Endpoint}

In the propensity matched analysis comparing exenatide BID and basal insulin, a significantly greater proportion of patients treated with exenatide BID reached the target of HbA1c $\leq 7.0 \%$ $(53 \mathrm{mmol} / \mathrm{mol})$ and weight loss. The respective proportions at the 6-month and 12-24-month time windows were $15.2 \%$ versus $6.2 \%$, and $18.4 \%$ versus $8.4 \%$. For the second target of $\mathrm{HbA} 1 \mathrm{c} \leq 7.0 \%(53 \mathrm{mmol} / \mathrm{mol})$ and minimum $5 \%$ weight loss, there was also a significantly greater proportion of patients achieving the target at each time window. The respective proportions were $10.0 \%$ versus $2.6 \%$ and $16.3 \%$ versus $5.2 \%$ at the 6 -month and $12-24$-month time windows (Table 5). 


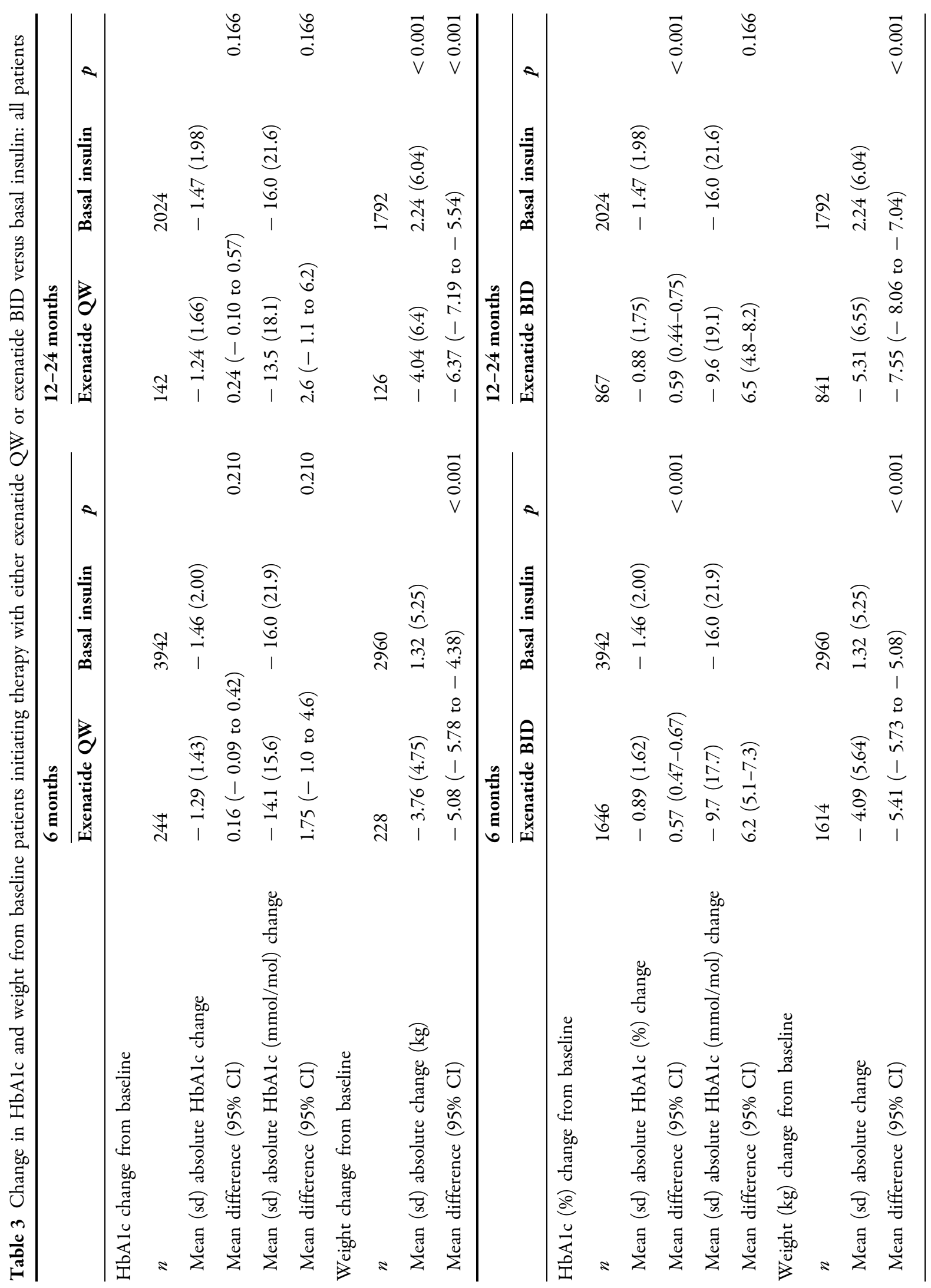


Table 4 Change in HbAlc and weight from baseline and proportion achieving combined glycemic and weight loss targets for patients initiating therapy with either exenatide QW or basal insulin: propensity matched patients

\begin{tabular}{|c|c|c|c|c|c|c|}
\hline & \multicolumn{3}{|l|}{6 months } & \multicolumn{3}{|c|}{ 12-24 months } \\
\hline & $\begin{array}{l}\text { Exenatide } \\
\text { QW }\end{array}$ & $\begin{array}{l}\text { Basal } \\
\text { insulin }\end{array}$ & $p$ & $\begin{array}{l}\text { Exenatide } \\
\text { QW }\end{array}$ & $\begin{array}{l}\text { Basal } \\
\text { insulin }\end{array}$ & $p$ \\
\hline \multicolumn{7}{|l|}{ HbAlc change from baseline } \\
\hline$n$ & 206 & 206 & & 111 & 111 & \\
\hline Female $[n(\%)]$ & $81(39.3 \%)$ & $91(44.2 \%)$ & & $39(35.1 \%)$ & $39(35.1 \%)$ & \\
\hline Baseline age [mean years, $(\mathrm{sd})]$ & $57.7(11.1)$ & $59.5(11.8)$ & & $57.9(10)$ & $58.5(11)$ & \\
\hline Baseline HbAlc \% [mean, (sd)] & $9.51(1.58)$ & $10.07(1.83)$ & & $9.51(1.71)$ & $9.83(1.62)$ & \\
\hline Baseline $\mathrm{HbAlc} \mathrm{mmol} / \mathrm{mol}[$ mean, (sd)] & $80.2(17.2)$ & $86.3(19.9)$ & & $80.2(18.6)$ & $83.7(17.7)$ & \\
\hline Mean (sd) absolute HbAlc \% change & $\begin{array}{r}-1.33 \\
(1.38)\end{array}$ & $\begin{aligned} &- 1.24 \\
&(2.01)\end{aligned}$ & & $\begin{array}{r}-1.19 \\
(1.73)\end{array}$ & $\begin{array}{r}-1.17 \\
(1.91)\end{array}$ & \\
\hline $\begin{array}{l}\text { Mean (sd) absolute } \mathrm{HbAlc} \mathrm{mmol} / \mathrm{mol} \\
\text { change }\end{array}$ & $\begin{aligned}- & 14.5 \\
& (15.0)\end{aligned}$ & $\begin{aligned}- & 13.5 \\
& (21.9)\end{aligned}$ & & $\begin{array}{r}-13.0 \\
(18.9)\end{array}$ & $\begin{array}{r}-12.8 \\
(20.8)\end{array}$ & \\
\hline Mean HbAlc \% difference (95\% CI) & \multicolumn{2}{|c|}{$-0.09(-0.43$ to 0.24$)$} & 0.45 & \multicolumn{2}{|c|}{$-0.02(-0.51$ to 0.46$)$} & 0.899 \\
\hline $\begin{array}{l}\text { Mean } \mathrm{HbAlc} \mathrm{mmol} / \mathrm{mol} \text { difference }(95 \% \\
\text { CI) }\end{array}$ & \multicolumn{2}{|c|}{$-1.00(-4.7$ to 2.5$)$} & 0.45 & \multicolumn{2}{|c|}{$-0.2(-5.6$ to 5.0$)$} & 0.899 \\
\hline \multicolumn{7}{|l|}{ Weight change from baseline } \\
\hline$n$ & 201 & 201 & & 100 & 100 & \\
\hline Female $[n(\%)]$ & $70(34.8 \%)$ & $69(34.3 \%)$ & & $37(37.0 \%)$ & $48(48.0 \%)$ & \\
\hline Baseline age [mean years, $(\mathrm{sd})]$ & $56.9(10.5)$ & $57.1(12.4)$ & & $58.6(10.2)$ & $59.4(11.2)$ & \\
\hline Baseline weight [mean $\mathrm{kg},(\mathrm{sd})]$ & $\begin{array}{l}110.67 \\
(19.9)\end{array}$ & $\begin{array}{l}107.99 \\
(22.16)\end{array}$ & & $\begin{array}{l}108.27 \\
(19.62)\end{array}$ & $\begin{array}{l}103.09 \\
(20.57)\end{array}$ & \\
\hline Mean (sd) absolute change (kg) & $\begin{aligned} &- 3.70 \\
&(4.42)\end{aligned}$ & $\begin{array}{l}1.20 \\
(5.72)\end{array}$ & & $\begin{array}{r}-3.24 \\
(5.32)\end{array}$ & $\begin{array}{l}2.48 \\
(6.3)\end{array}$ & \\
\hline Mean difference $(\mathrm{kg}, 95 \% \mathrm{CI})$ & \multicolumn{2}{|c|}{$-4.9(-5.9$ to -3.9$)$} & $<0.001$ & \multicolumn{2}{|c|}{$-5.73(-7.36$ to -4.1$)$} & $<0.001$ \\
\hline \multicolumn{7}{|l|}{ Patients reaching target } \\
\hline$n$ & 161 & 161 & & 88 & 88 & \\
\hline Female $[n(\%)]$ & $56(34.8 \%)$ & $53(32.9 \%)$ & & $32(36.4 \%)$ & $36(40.9 \%)$ & \\
\hline Baseline age [mean years, $(\mathrm{sd})]$ & $57.2(10.2)$ & $57.4(13.1)$ & & $57.7(10.9)$ & $56.3(12.3)$ & \\
\hline Baseline HbAlc \% [mean, (sd)] & $9.43(1.53)$ & $9.29(1.83)$ & & $9.53(1.64)$ & $9.53(1.71)$ & \\
\hline Baseline $\mathrm{HbAlc} \mathrm{mmol} / \mathrm{mol}$ [mean, (sd)] & $79.3(16.7)$ & $77.8(19.9)$ & & $80.4(17.9)$ & $80.4(18.6)$ & \\
\hline Baseline weight [mean, (sd)] & $\begin{array}{l}110.72 \\
(19.93)\end{array}$ & $\begin{array}{l}106.05 \\
(20.17)\end{array}$ & & $\begin{array}{l}107.13 \\
(18.83)\end{array}$ & $\begin{array}{l}103.51 \\
(18.06)\end{array}$ & \\
\hline $\begin{array}{l}\mathrm{HbAlc} \leq 7.0 \%(53 \mathrm{mmol} / \mathrm{mol}) \text { and any } \\
\text { weight loss }\end{array}$ & $36(22.4 \%)$ & $16(9.9 \%)$ & 0.002 & $16(18.2 \%)$ & $7(8.0 \%)$ & 0.044 \\
\hline
\end{tabular}


Table 4 continued

\begin{tabular}{|c|c|c|c|c|c|c|}
\hline & \multicolumn{3}{|l|}{6 months } & \multicolumn{3}{|c|}{ 12-24 months } \\
\hline & $\begin{array}{l}\text { Exenatide } \\
\text { QW }\end{array}$ & $\begin{array}{l}\text { Basal } \\
\text { insulin }\end{array}$ & $p$ & $\begin{array}{l}\text { Exenatide } \\
\text { QW }\end{array}$ & $\begin{array}{l}\text { Basal } \\
\text { insulin }\end{array}$ & $p$ \\
\hline $\begin{array}{l}\text { HbAlc } \leq 7.0 \%(53 \mathrm{mmol} / \mathrm{mol}) \text { and } \\
\text { weight loss } \geq 5 \%\end{array}$ & $19(11.8 \%)$ & $6(3.7 \%)$ & 0.007 & $7(8.0 \%)$ & $0(0 \%)$ & 0.007 \\
\hline
\end{tabular}

Table 5 Change in HbAlc and weight from baseline and proportion achieving combined glycemic and weight loss targets for patients initiating therapy with either exenatide BID or basal insulin: propensity matched

\begin{tabular}{|c|c|c|c|c|c|c|}
\hline & \multicolumn{3}{|l|}{6 months } & \multicolumn{3}{|c|}{ 12-24 months } \\
\hline & $\begin{array}{l}\text { Exenatide } \\
\text { BID }\end{array}$ & $\begin{array}{l}\text { Basal } \\
\text { insulin }\end{array}$ & $p$ & $\begin{array}{l}\text { Exenatide } \\
\text { BID }\end{array}$ & $\begin{array}{l}\text { Basal } \\
\text { insulin }\end{array}$ & $p$ \\
\hline \multicolumn{7}{|l|}{ HbAlc change from baseline } \\
\hline$n$ & 960 & 960 & & 411 & 411 & \\
\hline Female $[n(\%)]$ & $\begin{array}{l}425 \\
(44.3 \%)\end{array}$ & $\begin{array}{l}423 \\
(44.1 \%)\end{array}$ & & $\begin{array}{l}178 \\
(43.3 \%)\end{array}$ & $\begin{array}{l}173 \\
(42.1 \%)\end{array}$ & \\
\hline Baseline age [mean years, $(\mathrm{sd})]$ & $58.8(10.3)$ & $59.0(12.0)$ & & $58.9(9.7)$ & $58.8(11.9)$ & \\
\hline Baseline HbAlc [mean, (sd)] & $9.40(1.54)$ & $9.47(1.65)$ & & $9.32(1.55)$ & $9.35(1.71)$ & \\
\hline Baseline HbAlc mmol/mol [mean, (sd)] & $79.0(16.8)$ & $79.8(18.0)$ & & $78.2(16.9)$ & $78.5(18.6)$ & \\
\hline Mean (sd) absolute HbAlc change & $\begin{array}{r}-0.99 \\
(1.61)\end{array}$ & $\begin{array}{r}-1.04 \\
(1.71)\end{array}$ & & $\begin{array}{r}-1.03 \\
(1.64)\end{array}$ & $\begin{array}{r}-0.93 \\
(1.73)\end{array}$ & \\
\hline $\begin{array}{l}\text { Mean }(\mathrm{sd}) \mathrm{HbAlc} \mathrm{mmol} / \mathrm{mol} \text { change } \\
(95 \% \mathrm{CI})\end{array}$ & $\begin{array}{r}-10.8 \\
(17.5)\end{array}$ & $\begin{array}{r}-11.3 \\
(18.5)\end{array}$ & & $\begin{array}{r}-11.2 \\
(17.9)\end{array}$ & $\begin{array}{r}-10.1 \\
(18.6)\end{array}$ & \\
\hline Mean HbAlc \% difference (95\% CI) & \multicolumn{2}{|c|}{$0.05(-0.1$ to 0.2$)$} & 0.671 & \multicolumn{2}{|c|}{$-0.09(-0.32$ to 0.14$)$} & 0.095 \\
\hline $\begin{array}{l}\text { Mean } \mathrm{HbA} 1 \mathrm{c} \mathrm{mmol} / \mathrm{mol} \text { difference } \\
\quad(95 \% \mathrm{CI})\end{array}$ & \multicolumn{2}{|c|}{$0.5(-1.0$ to 2.0$)$} & 0.671 & \multicolumn{2}{|c|}{$-1.0(-3.5$ to 1.5$)$} & 0.095 \\
\hline \multicolumn{7}{|l|}{ Weight change from baseline } \\
\hline$n$ & 808 & 808 & & 458 & 458 & \\
\hline Female $[n(\%)]$ & $\begin{array}{l}357 \\
(44.2 \%)\end{array}$ & $\begin{array}{l}361 \\
\quad(44.7 \%)\end{array}$ & & $\begin{array}{l}194 \\
(42.4 \%)\end{array}$ & $\begin{array}{l}189 \\
(41.3 \%)\end{array}$ & \\
\hline Baseline age $[$ mean years, $(\mathrm{sd})]$ & $59.1(9.9)$ & $59.5(12.1)$ & & $58.8(9.9)$ & $58.4(11)$ & \\
\hline Baseline weight $[$ mean $\mathrm{kg}$, (sd)] & $\begin{array}{l}103.73 \\
(19.35)\end{array}$ & $\begin{array}{l}101.34 \\
(18.54)\end{array}$ & & $\begin{array}{l}103.42 \\
(18.22)\end{array}$ & $\begin{array}{l}101.43 \\
(17.16)\end{array}$ & \\
\hline Mean (sd) absolute change $(\mathrm{kg})$ & $\begin{array}{r}-3.46 \\
(5.15)\end{array}$ & $\begin{array}{l}0.82 \\
\quad(5.38)\end{array}$ & & $\begin{array}{r}-4.65 \\
(6.30)\end{array}$ & $\begin{array}{l}1.71 \\
\quad(6.378)\end{array}$ & \\
\hline Mean difference $(\mathrm{kg}, 95 \% \mathrm{CI})$ & \multicolumn{2}{|c|}{$\begin{array}{l}-4.28(-4.79 \text { to } \\
-3.76)\end{array}$} & $<0.001$ & \multicolumn{2}{|c|}{$\begin{array}{l}-6.37(-7.19 \text { to } \\
-5.54)\end{array}$} & $<0.001$ \\
\hline
\end{tabular}


Table 5 continued

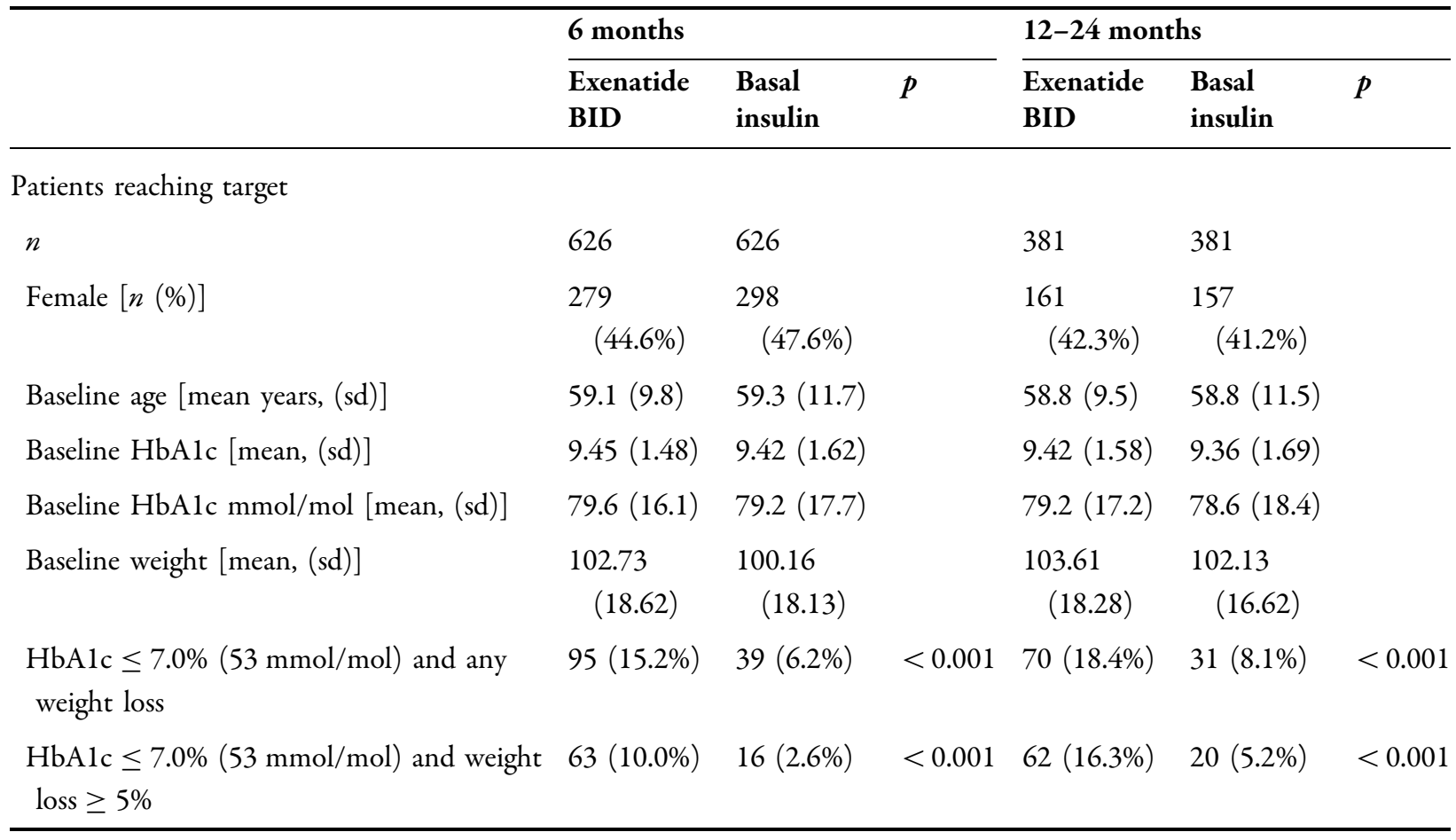

Table 6 Severe hypoglycaemic events, rates and comparable hazard ratio for patients initiating therapy with either exenatide QW or exenatide BID versus basal insulin: all patients and propensity matched patients

\begin{tabular}{|c|c|c|c|c|c|c|c|}
\hline & \multicolumn{2}{|c|}{ Exenatide } & \multicolumn{2}{|c|}{ Basal insulin } & \multirow[t]{2}{*}{ Hazard ratio } & \multirow[t]{2}{*}{$(95 \% \mathrm{CI})$} & \multirow{2}{*}{$p$} \\
\hline & Events & $\overline{\text { Rate }}$ & Events & $\overline{\text { Rate }}$ & & & \\
\hline \multicolumn{8}{|l|}{ EQW comparison } \\
\hline Unmatched & 0 & 0.0 & 167 & 15.5 & - & & \\
\hline Propensity matched & 0 & 0.0 & 1 & 3.6 & - & & \\
\hline \multicolumn{8}{|l|}{ EBID } \\
\hline Unmatched & 5 & 1.5 & 167 & 15.5 & 0.228 & $(0.092-0.570)$ & 0.002 \\
\hline Propensity matched & 5 & 2.2 & 17 & 7.4 & 0.369 & $(0.134-1.017)$ & 0.054 \\
\hline
\end{tabular}

\section{Exenatide BID Versus Basal Insulin: Time to Severe Hypoglycemia}

There were 5 (1.5 pky) severe hypoglycemic events recorded for patients treated with exenatide BID in the unmatched cohort compared with 167 (15.5 pky) in those treated with basal insulin; the hazard ratio was 0.228 (95\% CI 0.092-0.570). For those patients matched by propensity score the respective events were 5
(2.2 pky) versus 17 (7.4 pky) with an adjusted hazard ratio of 0.369 (95\% CI $0.134-1.017)$ (Table 6).

\section{DISCUSSION}

The treatment of diabetes emphasizes the maintenance of normoglycemia by either diet or pharmaceutical intervention. However, other factors are also important including the impact 
that different therapies can have upon a patient's weight profile. This study reports changes in both HbA1c and weight for patients with T2DM initiated with either exenatide or basal insulin as observed in routine data from UK primary care. In the propensity matched analyses, mean HbA1c fell in all treatment groups at each study time point with no significant difference observed between those treated with exenatide (either as QW or BID) and those treated with basal insulin. In the comparison of weight change, mean weight fell consistently in both exenatide QW and exenatide BID cohorts but mean weight increased in the basal insulin control groups. For the target endpoints, a significantly higher proportion of patients treated with exenatide, either as QW or BID, achieved both targets at both time points.

The reductions in HbA1c in both the exenatide and basal insulin cohorts observed in the propensity analyses were broadly similar to that observed in randomized clinical trial settings [16]. Although we did not report any significant difference between the study arms, Davies et al. [5] reported a significantly greater reduction in HbA1c for exenatide QW versus basal insulin detemir at 26 weeks with respective reductions in $\mathrm{HbA} 1 \mathrm{c}$ of $-1.30 \%$ and $-0.88 \%$. Similar reductions have also been reported when comparing exenatide BID with insulin glargine (respective decreases of $-1.25 \%$ and $-1.26 \%$ ) [7]. This reduction appears to be long-lasting as Diamant et al. [6] reported a statistically significant decrease in HbA1c at 3 years of $-1.01 \%$ and $-0.81 \%$ for patients treated with exenatide QW and insulin glargine, respectively.

A UK observational database study comparing glycemic and weight outcomes for patients prescribed GLP-1 versus insulin (glargine, detemir or NPH) also reported similar findings of a non-significant decrease in HbA1c and a significant difference in weight change favoring the GLP-1 cohort [17].

It is well documented that exenatide is associated with weight loss [18], whereas insulin leads to modest weight gain [19]. This was confirmed by our study which again supports findings from the clinical trials comparing exenatide with basal insulin. In a comparison of exenatide QW versus insulin detemir respective weight change of -2.7 and $+0.8 \mathrm{~kg}$ was reported, whereas in comparison of exenatide BID [4] with insulin glargine respective weight change at 26 weeks was $-2.73 \mathrm{~kg}$ compared with $+2.98 \mathrm{~kg}[6]$.

Two randomized trials considered a target HbA1c outcome combined with weight loss. On the basis of a target of $\mathrm{HbA} 1 \mathrm{C} \leq 7.0 \%$ (53 mmol $/ \mathrm{mol})$ and weight loss $\geq 1.0 \mathrm{~kg}, 44.1 \%$ patients treated with exenatide QW compared with $11.4 \%$ of determir treated patients achieved the target [5]. An earlier study considering a target of $\mathrm{HbA} 1 \mathrm{C} \leq 7.4 \%$ and weight loss $\geq 1.0 \mathrm{~kg}$ reported $53.4 \%$ of the exenatide group reaching target compared with $19.8 \%$ for the insulin glargine group [7].

This study therefore confirms that without compromising glycemic control, exenatide confers significant improvements in weight profile compared with basal insulin and that these findings transfer from trial settings to realworld practice. This may result in reduced healthcare costs as demonstrated in a companion study based on the same study cohorts in which we reported a significant reduction in healthcare utilization and associated costs [20].

As with all real-world studies based on routine data, there are certain limitations that must be considered in interpreting these results. Unlike a trial situation, patients were not randomized to particular treatments, thus confounding by indication should be considered as a potential factor. It is clear from the initial patient pools (Tables 1,2 ) that there are significant differences between those prescribed exenatide and those prescribed basal insulin at baseline. UK guidelines recommend the use of GLP-1 receptor agonists such as exenatide after the failure of triple therapy for patients with $\mathrm{BMI} \geq 35 \mathrm{~kg} / \mathrm{m}^{2}$ or for whom treatment with insulin would have significant occupational problems [21]. Consequently within this study, patients prescribed exenatide QW and exenatide BID had far higher BMI than those prescribed basal insulin (37.5 and 38.7 versus 29.9, respectively). The propensity matching process equalized the difference in baseline characteristics but it should be considered that those basal insulin patients who were matched by 
propensity score may therefore be considered atypical of the cohort as a whole and the generalizability of these results to the wider cohort should be considered. However, the results observed in the unmatched analysis were broadly similar to those observed in the propensity matched analyses for the basal insulin cohort. Patients included in the analyses also had to have remained on the index therapy at each time point. By definition therefore, patients who discontinued therapy quickly because of perceived lack of effectiveness or adverse events were excluded.

Within routine data, the HbA1c and weight outcomes were not collected systematically but were collected as part of the day-to-day patient administration. Patients with complete or nearcomplete data items may differ from those without. This may be due to the fact that they have more frequent consultations and thus greater chance for these parameters to be collected opportunistically or that they have characteristics which might increase the likelihood of such measurements being taken. A patient that appears overweight or obese, for example, may be more likely to have their weight recorded. As an example, in our study patients with weight recorded at 6 months in the EBID cohort had a mean baseline weight of $112.5 \mathrm{~kg}$ compared with $110.5 \mathrm{~kg}$ for those without a value recorded at 6 months.

Whilst we are able to determine the number of prescriptions issued we are not able to determine whether the prescriptions were collected or whether the patient was compliant with their regimen. However, the observation of reduced mean HbA1c in all cohorts may indicate compliance at a group level.

\section{CONCLUSION}

In this study we found that patients prescribed exenatide as either QW or BID formulations in real-world clinical practice had a decrease in mean HbA1c which was not significantly differently to that observed in those patients prescribed basal insulin. Moreover, these patients also had significant weight loss.

\section{ACKNOWLEDGEMENTS}

Funding. AstraZeneca funded both the study and the article processing charges.

Authorship. All named authors meet the International Committee of Medical Journal Editors (ICMJE) criteria for authorship for this manuscript, take responsibility for the integrity of the work as a whole, and have given final approval for the version to be published. Christopher Morgan helped design the study, performed the analysis, drafted the manuscript, and interpreted the results. Sara Jenkins-Jones performed the data extraction and manipulation, developed the concept of the study, and revised the manuscript. Craig Currie helped design the study, revised the manuscript, and interpreted the results. Sarah Holden developed the concept of the study, revised the manuscript, and interpreted the results. Qing Qiao helped design the study, revised the manuscript, and interpreted the results. Susan Grandy developed the concept of the study, revised the manuscript, and interpreted the results. Kristina Johnsson developed the concept of the study, revised the manuscript, and interpreted the results. All authors had full access to all of the data in this study and take complete responsibility for the integrity of the data and accuracy of the data analysis.

Disclosures. Qing Qiao is an employee of AstraZeneca. Susan Grandy is an employee of AstraZeneca. Kristina Johnsson is an employee of AstraZeneca, in which AstraZeneca commercialize exenatide. Christopher Morgan is an employee of Pharmatelligence. Sara JenkinsJones is an employee of Pharmatelligence. Craig Currie is an employee of Pharmatelligence. Sarah Holden is an employee of Pharmatelligence. Pharmatelligence received funding from AstraZeneca to conduct the study.

Compliance with Ethics Guidelines. This article is based on previously conducted studies and does not involve any new studies of human or animal subjects performed by any of the authors. 
Open Access. This article is distributed under the terms of the Creative Commons Attribution-NonCommercial 4.0 International License (http://creativecommons.org/licenses/ by-nc/4.0/), which permits any noncommercial use, distribution, and reproduction in any medium, provided you give appropriate credit to the original author(s) and the source, provide a link to the Creative Commons license, and indicate if changes were made.

\section{REFERENCES}

1. Inzucchi SE, Bergenstal RM, Buse JB, et al. Management of hyperglycaemia in type 2 diabetes: a patient-centered approach. Position statement of the American Diabetes Association (ADA) and the European Association for the Study of Diabetes (EASD). Diabetologia. 2012;55:1577-96.

2. Eberhart MS, Ogden C, Engelgau M, Cadwell B, Hedley AA, Saydah SH. Prevalence of Overweight and Obesity Among Adults with Diagnosed Diabetes-United States, 1988-1994 and 1999-2002. MMWR Morb Mortal Wkly Rep. 2004;53:1066-8.

3. Morgan CL, Jenkins-Jones S, Evans M, Barnett AH, Poole CD, Currie CJ. Weight change in people with type 2 diabetes: secular trends and the impact of alternative antihyperglycaemic drugs. Diabetes Obes Metab. 2012;14:424-32.

4. Kiiskinen U, Matthaei S, Reaney M, et al. Resource use and costs of exenatide bid or insulin in clinical practice: the European CHOICE study. Clin Outcomes Res. 2013;5:355-67.

5. Davies M, Heller S, Sreenan S, et al. Once-weekly exenatide versus once- or twice-daily insulin detemir: randomized, open-label, clinical trial of efficacy and safety in patients with type 2 diabetes treated with metformin alone or in combination with sulfonylureas. Diabetes Care. 2013;36:1368-76.

6. Diamant M, Van Gaal L, Guerci B, et al. Exenatide once weekly versus insulin glargine for type 2 diabetes (DURATION-3): 3-year results of an open-label randomised trial. Lancet Diabetes Endocrinol. 2014;42:464-73.

7. Davies MJ, Donnelly R, Barnett AH, Jones S, Nicolay C, Kilcoyne A. Exenatide compared with long-acting insulin to achieve glycaemic control with minimal weight gain in patients with type 2 diabetes: results of the helping evaluate exenatide in patients with diabetes compared with long-acting insulin (HEELA) study. Diabetes Obes Metab. 2009;11:1153-62.

8. Paul SK, Klein K, Magg D, Best JH. The association of the treatment with glucagon-like peptide-1 receptor agonist exenatide or insulin with cardiovascular outcomes in patients with type 2 diabetes: a retrospective observational study. Cardiovasc Diabetol. 2015;14:10.

9. Mogensen UM, Andersson C, Fosbøl EL, et al. Cardiovascular safety of combination therapies with incretin-based drugs and metformin compared with a combination of metformin and sulphonylurea in type 2 diabetes mellitus-a retrospective nationwide study. Diabetes Obes Metab. 2014;16:1001-8.

10. Potts JE, Gray LJ, Brady EM, Khunti K, Davies MJ, Bodicoat DH. The effect of glucagon-like peptide 1 receptor agonists on weight loss in type 2 diabetes: a systematic review and mixed treatment comparison meta-analysis. PLoS One. 2015;10(6): e0126769.

11. Heine RJ, Van Gaal LF, Johns D, Mihm MJ, Widel $\mathrm{MH}$, Brodows RG. Exenatide versus insulin glargine in patients with suboptimally controlled type 2 diabetes: a randomized trial. Ann Intern Med. 2005;143:559-69.

12. Holden SE, Jenkins-Jones S, Morgan CL, Schernthaner G, Currie CJ. Glucose-lowering with exogenous insulin monotherapy in type 2 diabetes: dose association with all-cause mortality, cardiovascular events and cancer. Diabetes Obes Metab. 2015;17:350-62.

13. Holman RR, Bethel MA, Mentz RJ, et al. Effects of once-weekly exenatide on cardiovascular outcomes in type 2 diabetes. $\mathrm{N}$ Engl $\mathrm{J}$ Med. 2017;377(13):1228-39.

14. Herrett E, Galllagher AM, Bhaskaran K, Forbes H, Mathur R, van Staa T, Smeeth L. Data resource profile: Clinical Practice Research Datalink (CPRD). Int J Epidemiol. 2015;44:827-36.

15. Charlson ME, Pompei P, Ales KL, MacKenzie CR. A new method of classifying prognostic comorbidity in longitudinal studies: development and validation. J Chronic Dis. 1987;40:373-83.

16. Busch RS, Ruggles J, Han J, Hardy E. Effects of exenatide twice daily, exenatide once weekly or insulin in patients with type 2 diabetes and baseline HbA1c $\geq 10.0 \%$ : two pooled analyses including 20 randomised controlled trials. Int J Clin Pract. 2017. https://doi.org/10.1111/ijcp.13029.

17. Hall GC, McMahon AD, Dain MP, Wang E, Home PD. Primary-care observational database study of 
the efficacy of GLP-1 receptor agonists and insulin in the UK. Diabet Med. 2013;30:681-6.

18. Vilsboll T, Christensen M, Junker AE, Gluud LL. Effects of glucagon-like peptide receptor agonists on weight loss: systematic review and meta-analyses of randomized controlled trials. BMJ. 2012;344:1-11.

19. Pontiroli AE, Miele L, Morabito A. Increase of body weight during the first year of intensive insulin treatment in type 2 diabetes: systematic review and meta-analysis. Diabetes Obes Metab. 2011;13:1008-19.
20. Holden SE, Morgan CL, Qiao Q, Jenkins-Jones S, Berni ER, Currie CJ. Healthcare resource utilization and related financial costs associated with glucose lowering with either exenatide or basal insulin: a retrospective cohort study. Diabetes Obes Metab. 2017;19:1097-105.

21. National Institute for Health and Care Excellence. Type 2 diabetes in adults: management. NICE Guidelines [NG28]. 2015. https://www.nice.org.uk/ guidance/ng28. Accessed 05 Jan 2017. 